\title{
Reducción y Fijación de Fractura Orbitomalar con Malla de Titanio. Reporte de un caso y revisión de la literatura.
}

Fracture Reduction and Fixation orbitomalarç with titanium mesh. Case Report and review of the li terature.
Fratura Redução e orbitomalar Fixation com tela de titânio. Relato de Caso e revisão da literatura.
Fecha de Recepción

02 de octubre de 2012
Aceptado para su publicación

II de diciembre de 2012

\section{Roque Oscar Rosende}

Prof. Titular. Cirugía I Técnicas quirúrgicas y anestésicas.

\section{Estefanía Raquel Fernández}

Od. Becaria de Investigación en Clínica Estomatológica.

Sebastián Krupp

Od. Responsable del Servicio de Cirugía Buco-Maxilofacial UNNE.

\section{Resumen}

Las fracturas del complejo orbitomalar representan el $13 \%$ de las fracturas craneofaciales, siendo únicamente más frecuentes las fracturas de los huesos propios nasales. Las cavidades orbitarias son dos extensas depresiones o fosas destinadas al globo ocular y sus anexos, presentan una forma de pirámide cuadrilátera. En el caso de una fractura orbitomalar o de piso de órbita, no siempre afecta los rebordes orbitarios, siendo el mayor problema la caída del tejido periorbitario hacia el seno maxilar con el consecuente descenso del globo ocular. Entre las causas más frecuentes de este tipo de fracturas están las agresiones, seguidas por los accidentes de tráfico, caídas y accidentes deportivos. Este trabajo tiene como objetivo aportar información actualizada sobre las fracturas orbitomalares en cuanto a su etiología, diagnóstico clínico e imagenológico y tratamiento a seguir, para luego abordar un caso clínico de un paciente de 20 años que se presentó a la consulta en el Hospital Odontológico de la Facultad de Odontología de la Universidad Nacional del Nordeste con un traumatismo a nivel del tercio medio de un lado de la cara reconocido por los especialistas como una fractura orbitomalar. A partir de su diagnóstico se propuso un plan de tratamiento quirúrgico con la posterior colocación de una malla de titanio que actuase como fijación de los segmentos óseos.

\section{Pallabras Claves}

Fractura orbitomalar, trauma facial, traumatismo orbitocigomático, piso de órbita, malla de titanio. 


\section{Introducción}

Las fracturas del complejo orbitomalar, por su peculiar posición prominente en la cara, constituyen una patología común para el cirujano oral y maxilofacial. El hueso malar es el parachoques lateral medio facial y forma parte del suelo y de la pared lateral orbitaria. Entre sus funciones se encuentran: proteger al globo ocular al dar proyección a la zona del pómulo, dar inserción al músculo masetero y absorber la fuerza de cualquier impacto antes de que éste afecte al encéfalo. A la importante función de parachoques medio-facial hay que añadir una importante trascendencia en la estética facial (pómulos prominentes).

En las fracturas orbitomalares podemos encontrar afectación de sus cuatro arbotantes clásicos, que son: su articulación con el maxilar (maxilomalares: apof. piramidal y apof. ascendente del maxilar-RIO-), con el hueso frontal (frontomalar), y con el temporal (cigomático-malar). En la actualidad se considera un quinto arbotante en su unión con el ala mayor del esfenoides (pared lateral orbitaria). El patrón de fractura más frecuente es aquel que recorre las suturas naturales del cigoma con el resto de huesos con que se articula.

Considerando que su abordaje quirúrgico responde a las necesidades del paciente, éstas se relacionaran con las premisas de estética y función señaladas por el paciente. De esta forma, alteraciones visuales y oclusales suelen ser frecuentes demandas de función, mientras que la pérdida de proyección anteroposterior, rotaciones óseas y conminuciones en fractura con pérdida de soporte de tejido blando es asociada a las demandas estéticas en el paciente traumatizado según las consideraciones de Ellis y Kittidumkerng, 1996'.

En cuanto a los factores etiológicos podemos mencionar en primer lugar a las agresiones con traumatismo directo en la región orbitomalar, así como accidente de circulación, traumatismos faciales por caídas casuales y accidentes deportivos.

La exploración clínica de los pacientes que han sufrido traumatismo orbitocigomático puede verse muy dificultada por la presencia de edema y hematoma de los tejidos periorbitarios que en numerosas ocasiones puede enmascarar la existencia de fracturas subyacentes.
Clínicamente en las fracturas del complejo orbitocigomático podemos encontrar los siguientes signos y síntomas como son edema y hematoma periorbitario como moretones en el surco vestibular bucal superior en el lado afectado, equimosis subconjuntival y escleral, desviación antimongoloide de la hendidura palpebral, que se acompaña de acortamiento de la altura del párpado inferior y exposición escleral, trismus mecánico como consecuencia de un impedimento a nivel de la apófisis coronoides por el desplazamiento del hueso cigomático, distopía ocular y/o enoftalmos traumático o exoftalmos y proptosis del ojo, diplopía por restricción de la motilidad ocular extrínseca, aumento de la anchura facial, asimetría facial por hundimiento del pómulo, hipoestesia de región malar, párpado inferior y fosa nasal ipsilateral por afectación del nervio infraorbitario, dolor. Otros signos para tener en cuenta son enfisema subcutáneo, en caso que la fractura comunique la órbita con zonas aireadas como el seno maxilar o las fosas nasales, fácilmente identificable por la inflamación súbita y la crepitación de la zona al palpar y deformidad del arco cigomático.

Los enoftalmos, así como los informes de diplopía, son indicativos de la necesidad de una intervención quirúrgica. Otra indicación es la hernia de tejido periorbital a través de defectos en el suelo y la presencia de fragmentos de hueso dentro de la órbita demostrado por estudios de imagen. Para el diagnóstico de esta lesión es necesario someter al paciente a una serie de pruebas de exploración como son la ocular teniendo en cuenta la agudeza visual, campo visual, percepción de la luz, reactividad pupilar mediante los reflejos fotomotor directo y consensual, fondo de ojo, motilidad ocular extrínseca. Posteriormente, un examen facial completo contemplando palpación bilateral comparando ambos RIO, suturas frontomalares, suturas maxilomalares, rebordes orbitarios externos, cuerpo malar y arco cigomático buscando escalones o asimetrías óseas. Para concretar el examen clínico es de vital importancia considerar la exploración intraoral a nivel de la unión maxilomalar².

Como diagnóstico por imágenes el mejor recurso utilizado en laactualidad esla Resonancia Magnética Nuclear (RMN) de alta resolución que permite la visualización tanto de tejidos duros como blandos en diferentes cortes. Acompañado de estudios 
radiográficos complementarios como pueden ser la Proyección de Waters, Proyección modificada de Waters (fronto-occipital PA), Proyección de Hirtz, Técnica de Caldwell, Radiogradía oblicua de cráneo-cara, Proyección de Rhese como así también son de gran utilidad la Tomografía computarizada (TC) y la ecografía orbitaria.

Una vez establecido el diagnóstico certero se procede con el manejo terapéutico que dependerá de ciertos factores a mencionar, como tipo y cantidad de desplazamiento de la fractura, grado de conminución de los fragmentos, sintomatología asociada (diplopía, enoftalmos, dificultad de apertura oral), patología sistémica del paciente que pueda comprometer la anestesia y/o favorecer complicaciones del manejo anestésico, durante y después de la intervención, condiciones locales y/o sistémicas que puedan comprometer la hemostasia durante o después de la cirugía, factores anatómicos locales que puedan incrementar el riesgo de morbilidad, secuelas de cirugías o lesiones previas, anormalidades funcionales o de desarrollo de los tejidos blandos faciales y capacidad de entendimiento del paciente y acompañantes de su problema y su corrección.

A través del tratamiento propuesto al paciente se pretende normalizar la función de las estructuras faciales, restaurar la estructura tridimensional del complejo orbitomalar actuando a nivel del ancho, proyección y altura facial, así como la simetría facial. Restaurar el volumen orbitario para evitar la distopía y el enoftalmos, eliminar la sintomatología potencialmente atribuible a la fractura orbitomalar, optimizar la salud futura de las estructuras faciales, conseguir una estética facial óptima, obtener resultados estables a largo plazo, minimizar la morbilidad asociada al tratamiento, satisfacer las expectativas del paciente, reducir el tiempo total del tratamiento y emplear los recursos disponibles de la manera más eficiente posible.

Cuando se propone un plan de tratamiento, éste debe estar acorde en primer lugar a los grados y dirección del desplazamiento de los cabos de fractura, inestabilidad de los fragmentos, si se encuentra en presencia de una fractura conminuta y signos clínicos que presente el paciente como consecuencia de dicho trauma. Como pueden ser:

Signos Oculares. La disrupción del piso orbitario se puede presentar juntamente con las fracturas cigomáticas o maxilares o puede existir también como una lesión separada. Esto último se produce por la transmisión de un aumento de la presión intraorbitaria que ocurre, por ejemplo, cuando un puño golpea los tejidos blandos de la órbita.

En las fracturas del suelo de la órbita se produce la pérdida de soporte por parte de los tejidos permitiendo que el globo ocular quede situado más profundamente en el interior de la órbita produciéndose clínicamente un enoftalmos. Este signo clínico rara vez se observa inmediatamente después de las fracturas, pues queda compensado por la tumefacción del contenido orbitario, debido principalmente a la hemorragia que se produce entre los tejidos blandos. Normalmente, el enoftalmos se observa sólo cuando remite la tumefacción.

Entre los signos más molestos que aparecen después de las fracturas cigomáticas, está la diplopía. La causa de la diplopía es la interferencia con uno o más músculos extraoculares que producen una imposibilidad de mover los ojos sincrónicamente. Barclay recoge 4 causas de inactividad muscular: I) laceración del músculo; 2) reflejo inhibitorio del músculo; 3) separación de la inervación y 4) pinzamiento muscular. Las dos primeras causas, juntamente con el edema, son muchas veces la causa de la diplopía temporal que se observa en los pacientes con traumatismo facial dentro o alrededor de la órbita. El tiempo de recuperación suele ser de 5 días, pero en casos excepcionales pueden necesitarse 10 a 14 días.

Si se afectan los músculos de la zona de fracturas, se produce un desequilibrio vertical y el ojo afectado queda relativamente fijo en comparación con el lado libre. El ojo puede quedar fijo en una posición deprimida o elevada (hipotropía o hipertropía), dependiendo de donde el músculo ha sido atrapado. Normalmente, la complicación que se observa con más frecuencia es el pinzamiento del recto inferior anterior al ecuador del globo (con la correspondiente depresión del ojo en el lado afectado).

Signos Bucales. Entre ellos la dificultad para la apertura oral es el de mayor incidencia.

Signos Faciales. Se puede observar deformidad facial post-traumática, asimetría, hundimiento del hueso cigomático, deformación del arco cigomático.

La reducción de la fractura del piso de orbita se 
acompaña con algún procedimiento reconstructivo, que puede ser con injertos óseos o más frecuentemente con malla de titanio. La extensión de la exploración del piso orbitario debe ser cuidadosa; siempre existe la posibilidad de desencadenar el reflejo óculo-cardíaco o en casos complejos, producir alguna secuela nerviosa.

Posteriormente, se procede con la utilización de una malla de titanio, dicho material es el más utilizado hoy en día en las fracturas cráneo-faciales, teniendo en cuenta los factores que influyen en su elección, tales como el tamaño del defecto, la participación de múltiples paredes de la órbita, la adaptación a la órbita del contorno, la restauración del volumen adecuado, la prevención de la migración y el desplazamiento mediante la fijación de los materiales con suturas, tornillos o adhesivos, el riesgo de adherencias y la restricción de la motilidad ocular, la prevención de la colonización bacteriana y el momento de la corrección en relación con trauma.

Las mallas de titanio son implantes metálicos que han revolucionado el tratamiento de las fracturas faciales, lo que permite una fijación estable de la forma tridimensional. Estos materiales son ampliamente aceptados y ofrecen variadas opciones terapéuticas; por ejemplo, cuando se presentan fracturas blow out, la malla de titanio presenta buenos resultados, aparentemente mejores que el injerto óseo desde que no conlleva aumento de volumen ${ }^{3}$; la espesura de la malla de titanio está entre 0,15 y $0,20 \mathrm{~mm}$. Esta tecnología ha contribuido a lograr una reparación anatómica precisa, muy favorable a largo plazo, con una reducción de las complicaciones relacionadas con el cambio de volumen y el ojo, reduciendo el riesgo de enoftalmos. No obstante, no se descarta una de las preocupaciones inherentes al uso de este material que es el riesgo de una respuesta inflamatoria local, con la formación de adherencias que pueden contribuir a la restricción del estrabismo.

Sin embargo, existe una línea de trabajo que estipula, en casos de trauma complejo de órbita, el uso de más de un material, conjugando los injertos óseos y los materiales aloplásticos como malla de titanio ${ }^{4}$.

\section{Caso Clínico}

Paciente masculino de 18 años de edad procedente de la Provincia de Corrientes - Argentina, que acude al servicio del Hospital Odontológico de la Facultad de Odontología de la Universidad Nacional del Nordeste (UNNE), refiriendo como motivo de consulta trauma facial en región orbitomalar.

\section{Historia clínica}

Antecedentes personales: el paciente relató no padecer ninguna enfermedad en particular.

Examen clínico: paciente sexo masculino de 18 años de edad concurre a la consulta por trauma facial. Paciente estable, lúcido, orientado en tiempo y espacio, afebril, asintomático.

Al examen clínico presenta: edema en la región antero superior de la cara, hematoma palpebral y equimosis subconjuntival.

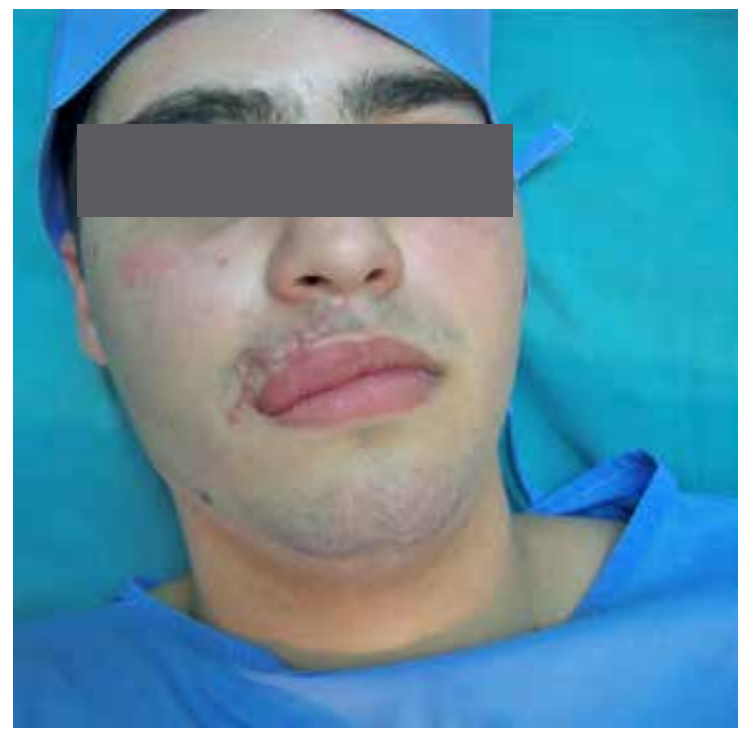

Figura I. Examen clínico prequirúrgico del paciente a intervenir.

Diagnóstico y pronóstico: Al observar la TAC (Tomografía Axial Computada) se evidencia que no existe fractura del piso de la órbita como se observa en la figura 2. Por el contrario, se comprueba una extravasación de material sanguíneo en el seno maxilar derecho proveniente de la fractura a nivel del reborde maxilar y piezas dentarias 15 y 16. 


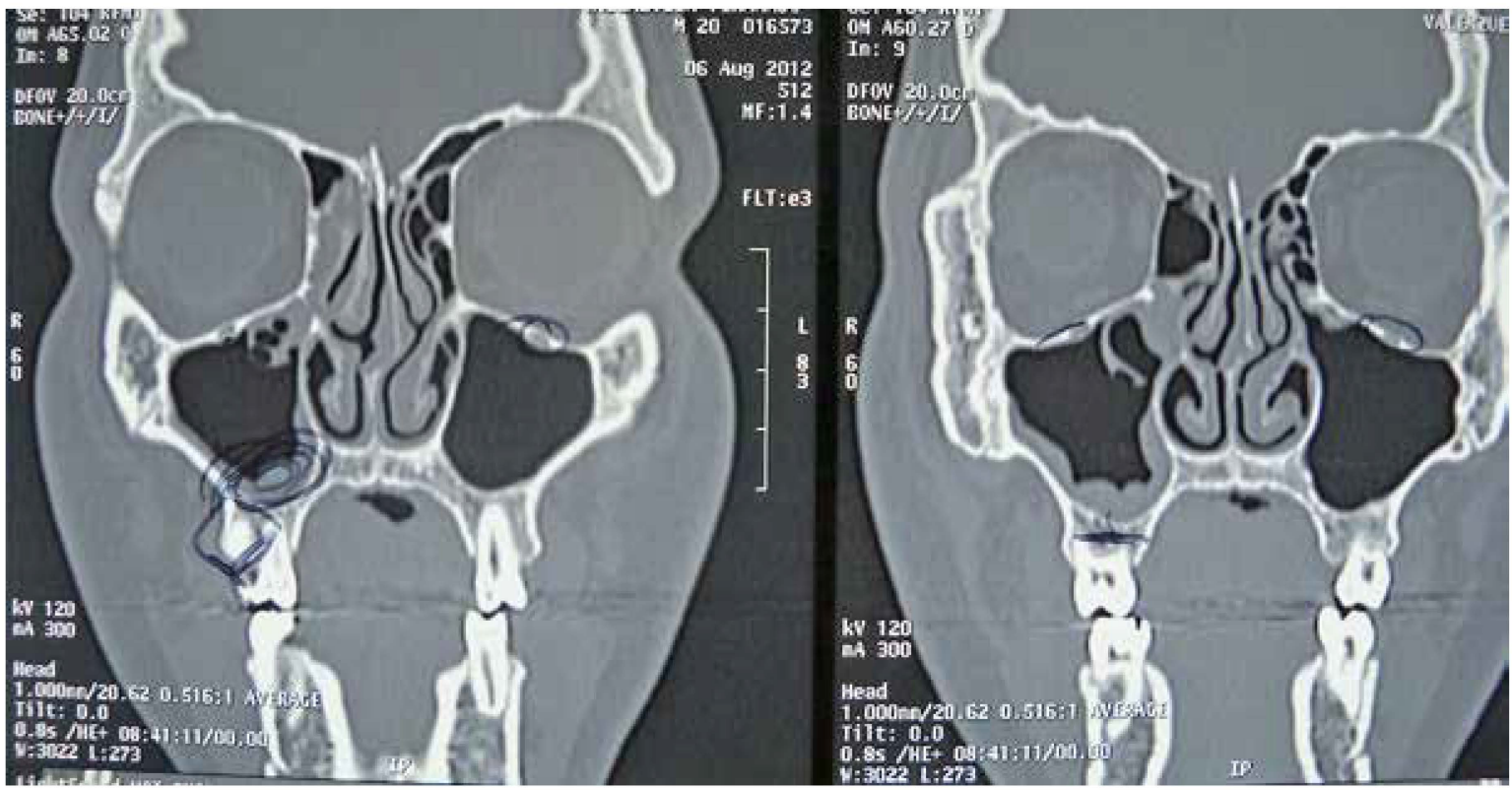

Figura 2. Tomografia Axial Computada. Corte Coronal.

En la figura 3 se observa en el interior del seno maxilar derecho parte de la grasa periorbitaria a través de un corte axial de la tomografía.

El pronóstico del caso es bueno siguiendo el protocolo de tratamiento.

Tratamiento: se pidió al paciente que se realizara los exámenes clínicos prequirúrgicos, para tener conocimiento acerca del estado general del mismo previo a la cirugía.

En las sucesivas consultas del paciente se pres- cribió la medicación prequirúrgica dentro de la cual se encontraban antibióticos para reducir el riesgo de infección postoperatoria, corticoides para reducir el edema y el dolor postoperatorio y antiinflamatorios no esteroideos (AINES), tras el empleo de corticoides, para reducir el dolor y la tumefacción.

El paciente fue intervenido en la sala de quirófano de la Clínica Medicis de la ciudad de Corrientes - Capital. Se inició el procedimiento quirúrgico

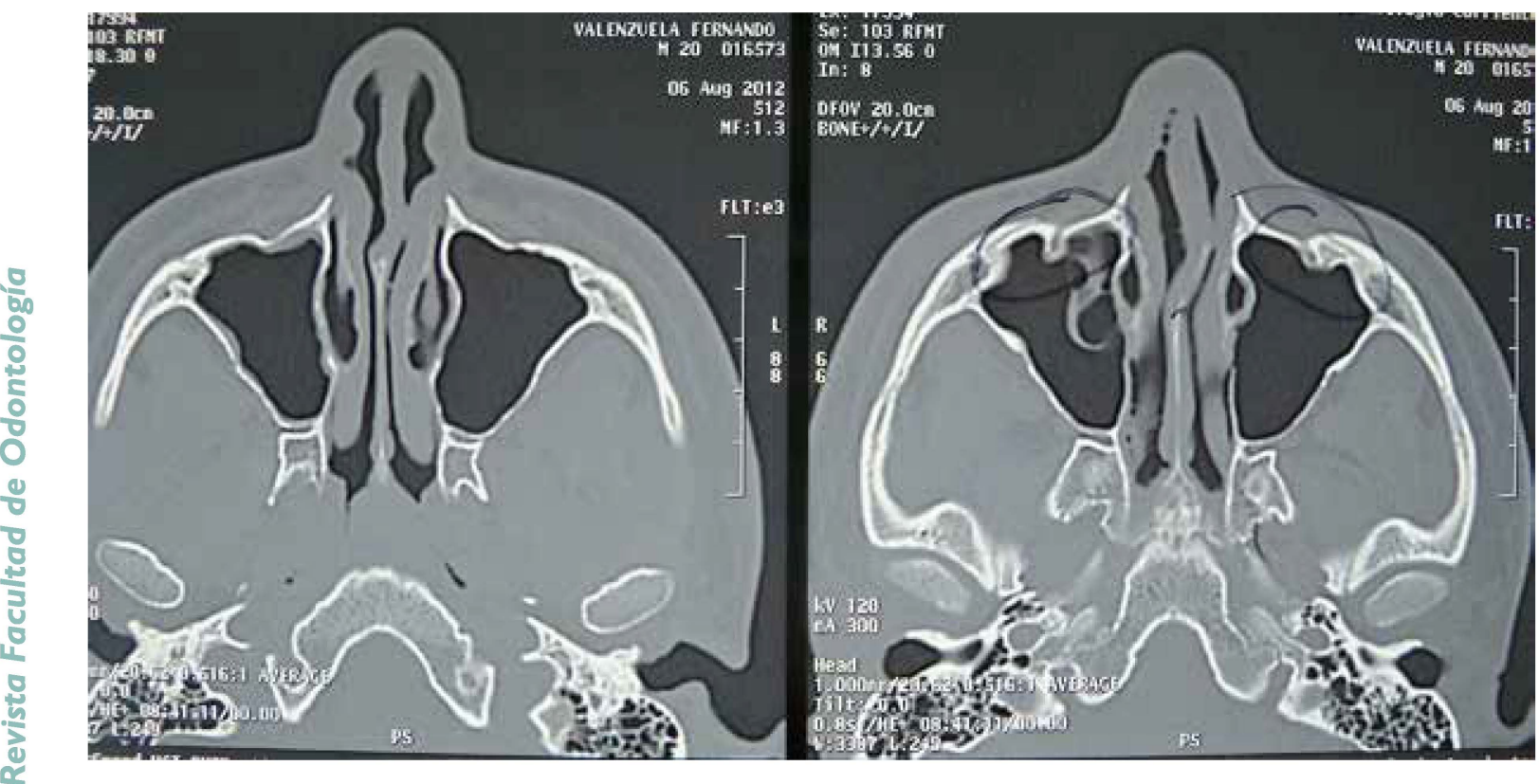

Figura 3. Tomografia Axial Computada. Corte Axial. 


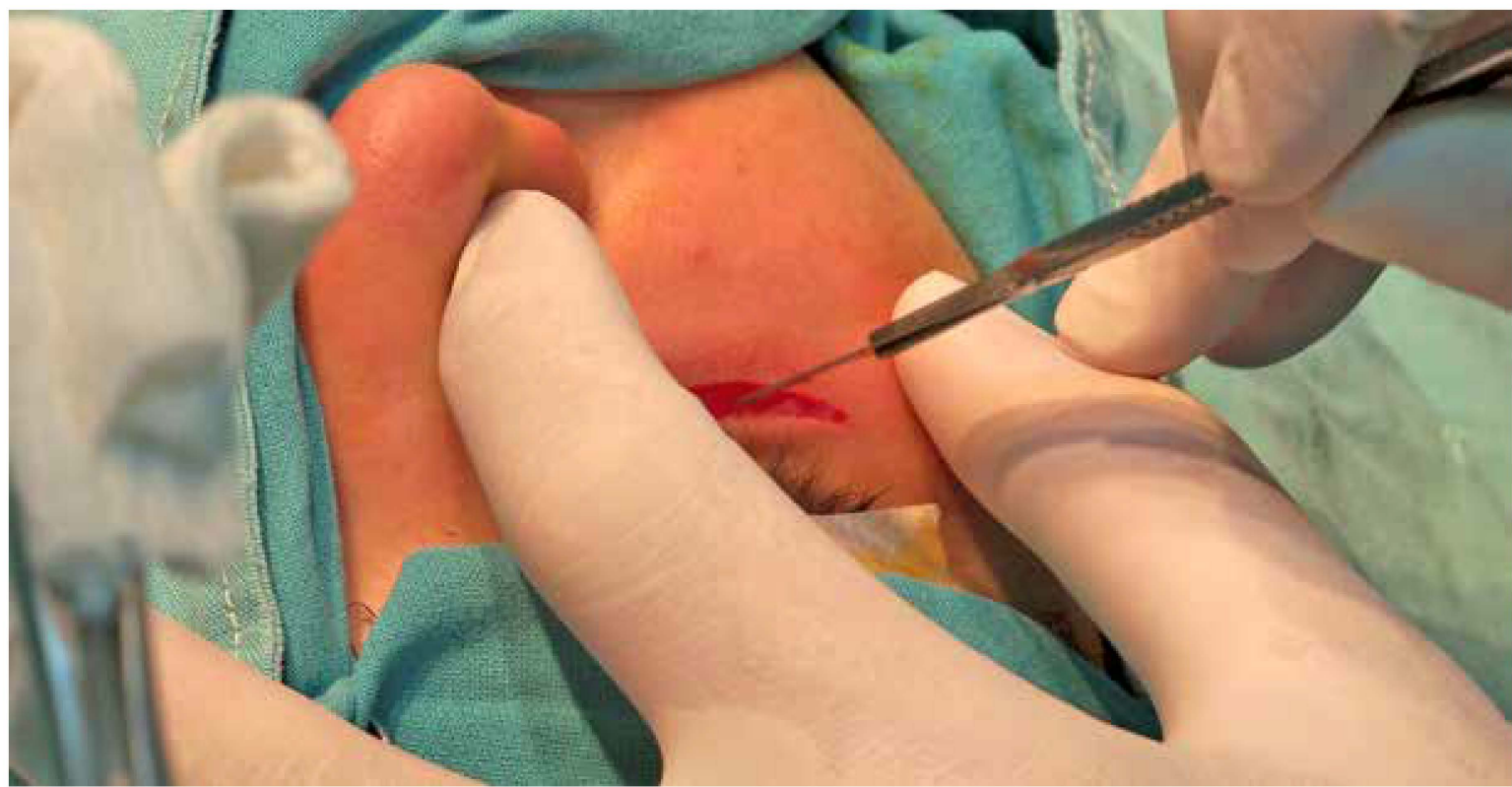

Figura 4. Acceso subciliar para reborde infraorbitario y piso de órbita.

bajo anestesia general con intubación nasotraqueal. Luego se procedió con la preparación del paciente y del campo quirúrgico.

La técnica quirúrgica empleada para el abordaje fue una incisión externa del párpado inferior para permitir abordar el borde infraorbitario y piso orbitario, denominada incisión subciliar, también conocida como incisión infraciliar o blefaroplastia. Esta incisión se hace por debajo de las pestañas, cuya ventaja radica en una cicatriz imperceptible y la facilidad de extender la incisión para una exposición adicional de todo el borde de la órbita lateral en caso que fuera necesario ${ }^{5}$.

Abordado el foco de fractura, el piso de la órbita se revisó con cuidado mediante una legra, levantando el periostio hasta encontrar la línea de fractura o eventualmente la rotura de éste; en caso de que se hubiese presentado este último, se presentaría una amplia apertura hacia el seno maxilar ${ }^{6}$. Seguidamente se realizó una reducción abierta y exposición de todos los focos de fractura implicados.

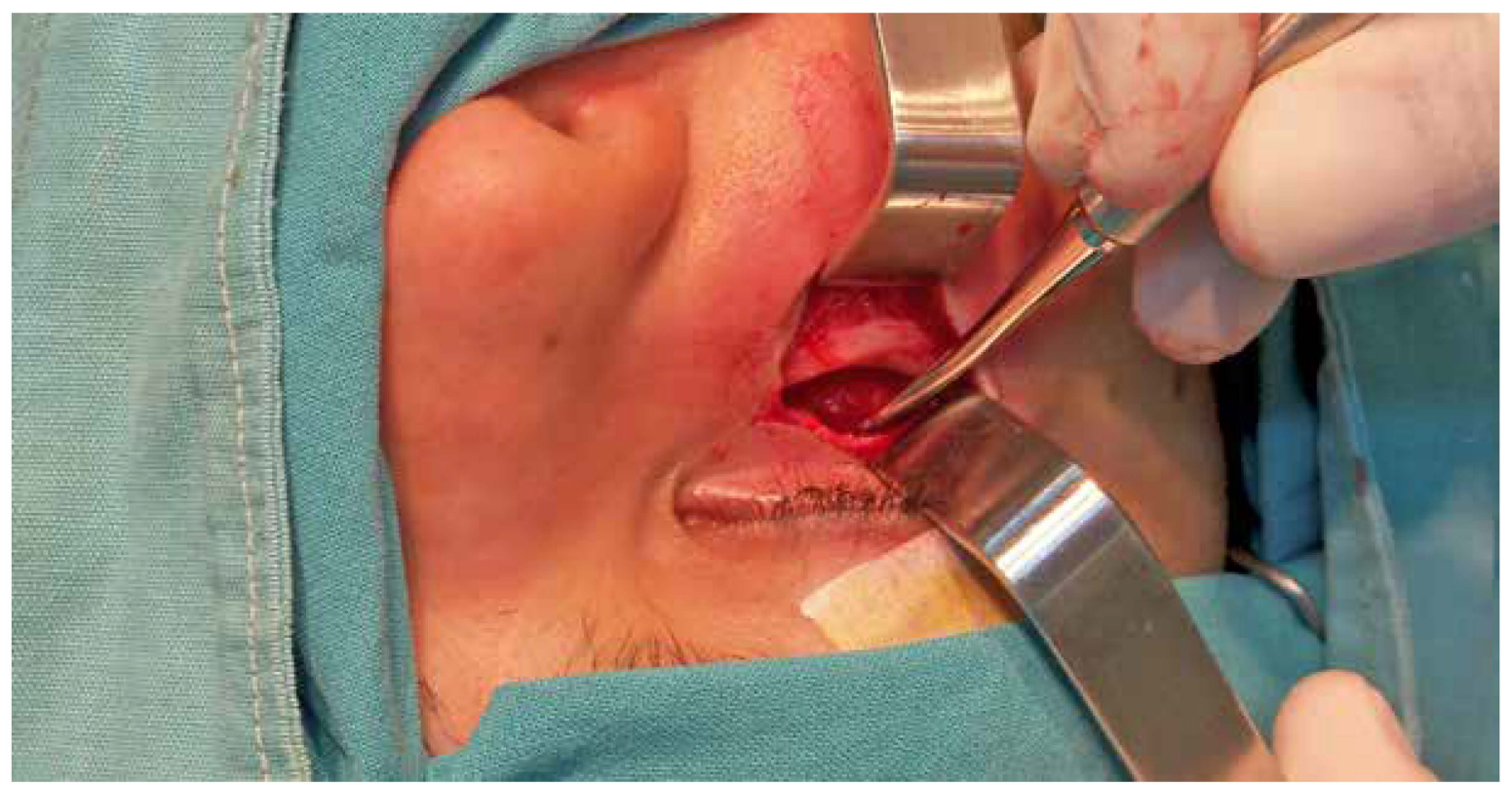

Figura 5. Abordaje del piso de la órbita. 


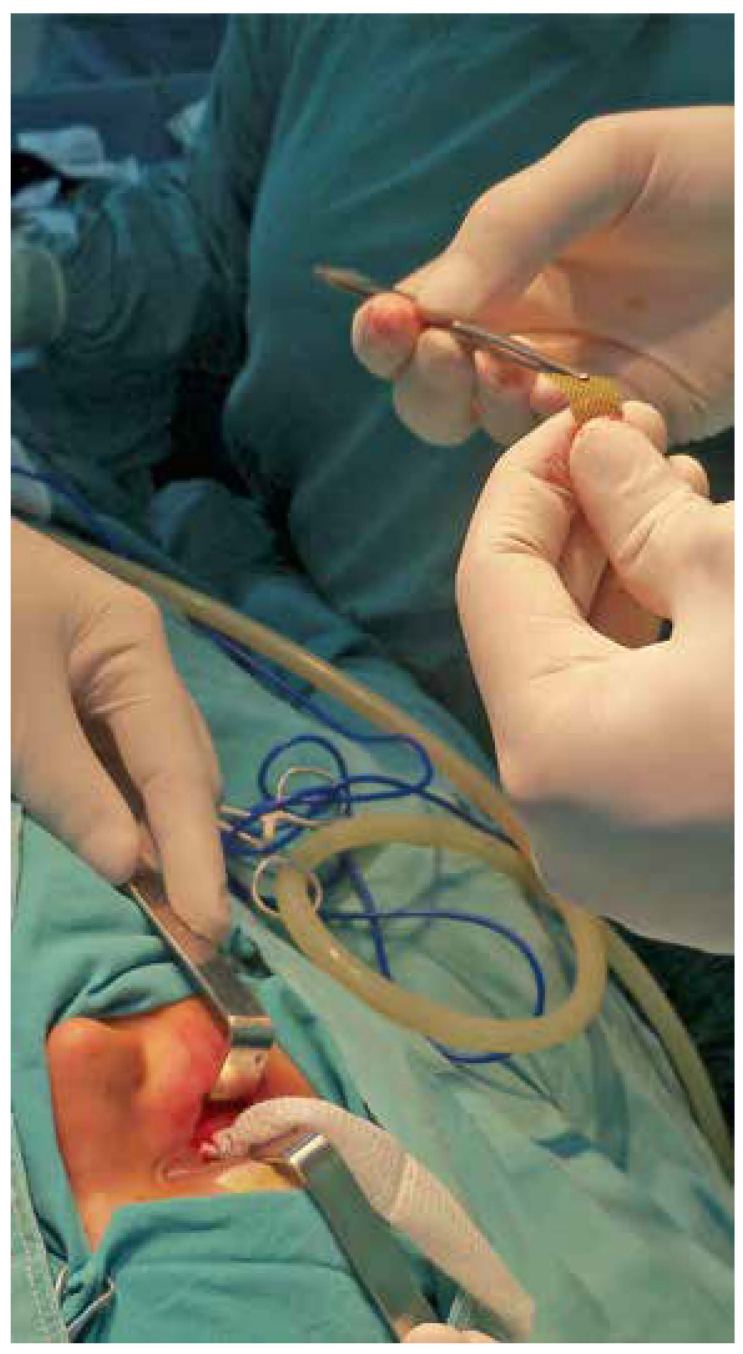

La reducción y fijación anatómica impiden los desplazamientos secundarios y permite una osificación primaria de la fractura. Para conseguir gran estabilidad, buen contacto óseo y una adecuada formación del callo de fractura se optó por la utilización de miniplacas o microplacas de titanio de $1,5 \mathrm{~mm}$ siendo superior a la osteosíntesis con alambres. Aproximadamente un tercio de las fracturas cigomáticas requieren cierto grado de fijación después de la reducción?

Una precaución a tener en cuenta es que los músculos extraorales no queden pinzados por el elemento colocado y que el suelo de la órbita no resulte "sobrerreducido". Se ha descrito un caso de ceguera producida por la penetración de fragmentos óseos en el interior del suelo de la órbita y la consiguiente lesión del nervio óptico. Una vez que se reparó el traumatismo causante de la lesión de la órbita, se practicaron las pruebas de movilización forzada de los globos oculares. Estas pruebas consisten en sujetar delicadamente los músculos extraoculares a través de la conjuntiva con un pequeño fórceps o pinza dentada y girar el globo en todas las direcciones. Si un músculo queda trabado en un punto de fractura, se observará que existe una dificultad en el giro del globo ocular en la dirección de este músculo. El paciente fue mantenido en observación en el

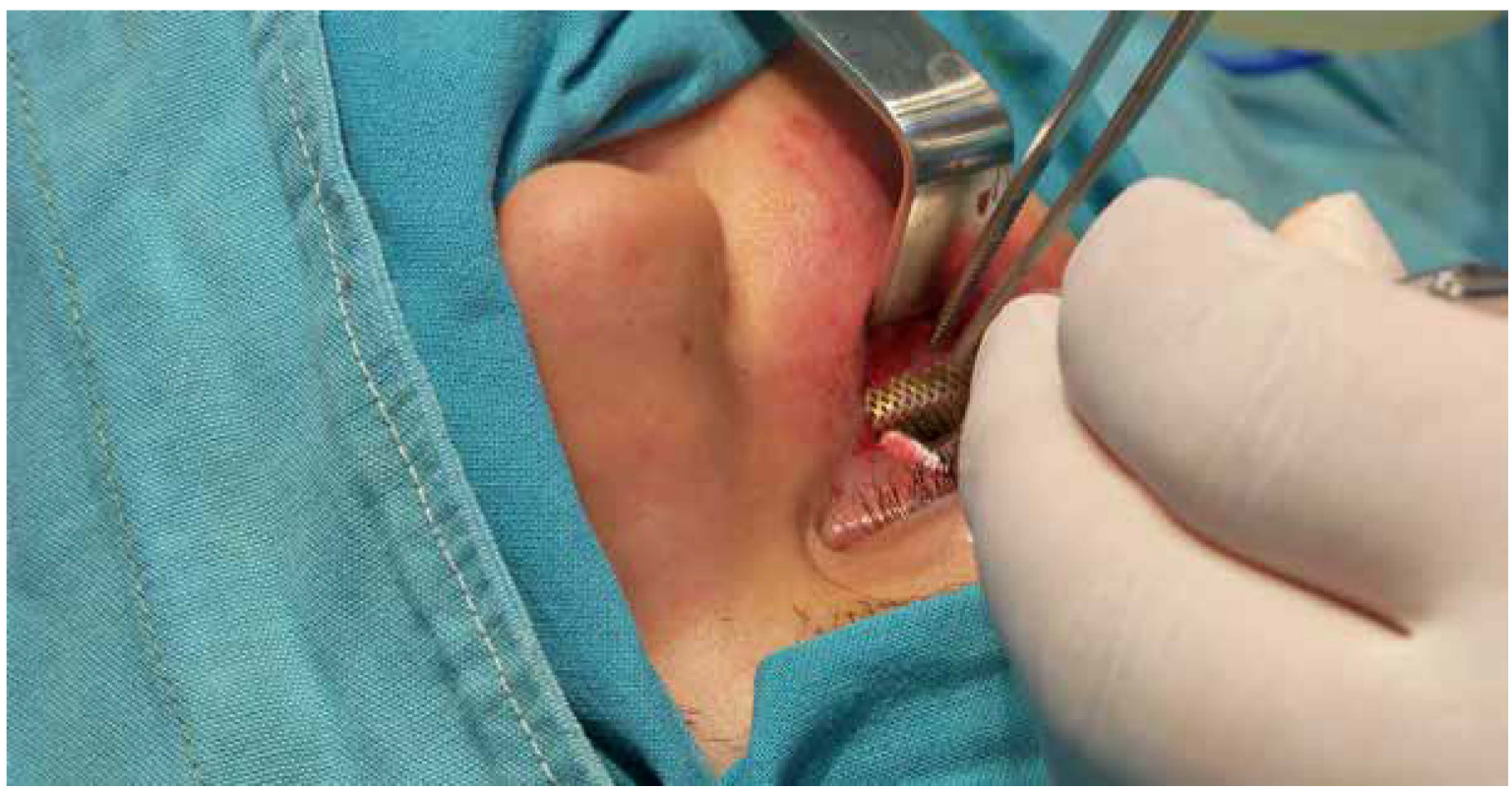

Figura 6. Colocación de la malla de titanio de un espesor de $0,15 \mathrm{~mm}$. Estas son delgadas, lo que permite el ajuste y modelaje fácilmente, de modo que puede adaptarse al contorno del piso de la órbita y promover el apoyo eficaz de grandes defectos. 


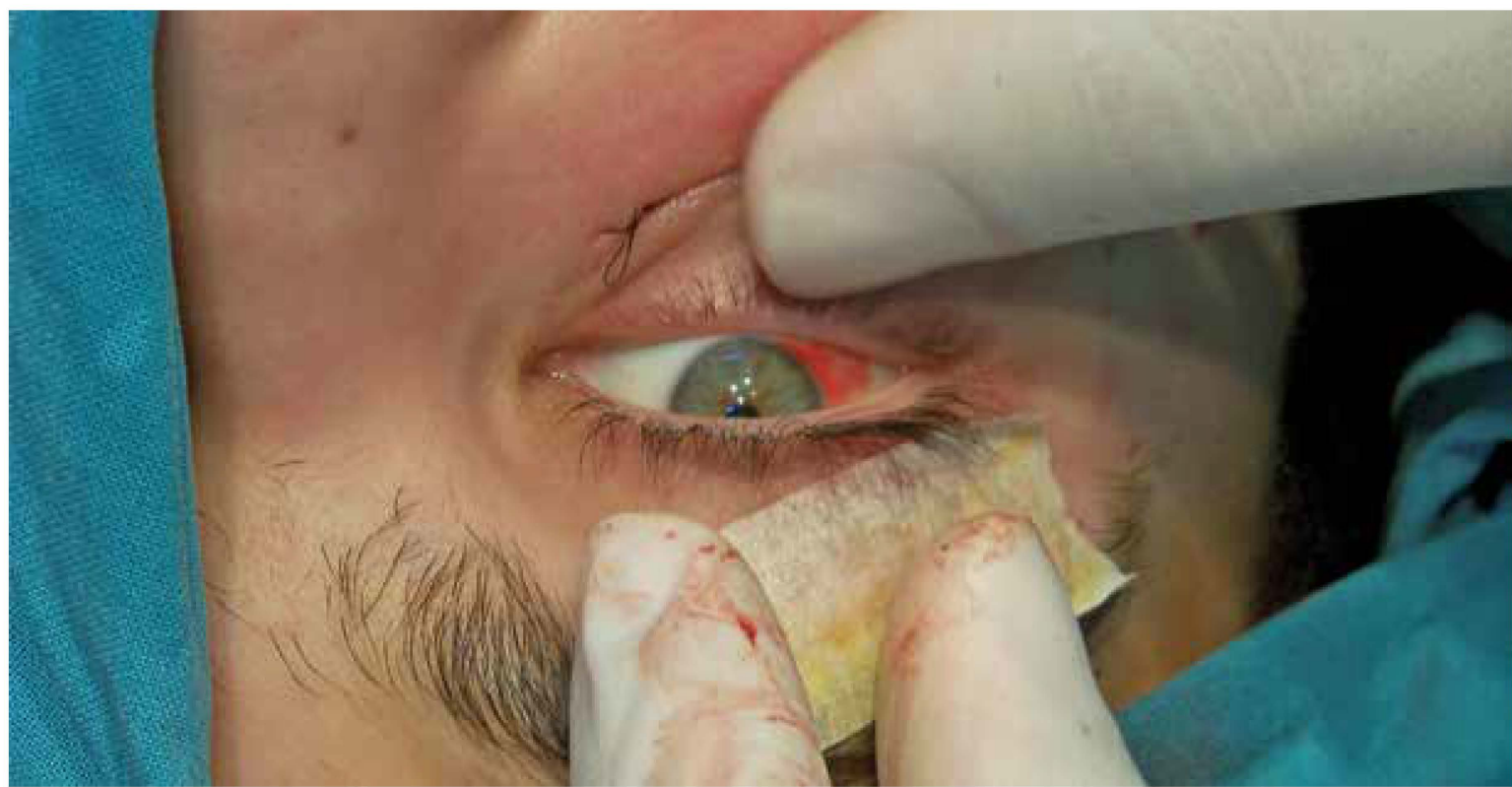

Figura 7. Las pruebas de conducción forzada consisten en asir los músculos extraoculares y asegurarse de que el globo se puede movilizar en todas las direcciones.

hospital por $\mathbf{2 4}$ horas donde se efectuaron las revisiones postoperatorias para evaluar la evolución del paciente insistiendo sobre todo en la función ocular. Todos los indicadores fueron de resultado favorable con obtención de los objetivos terapéuticos prefijados y ausencia de sintomatología atribuible a la fractura orbitocigomática. Sólo se observó inflamación de la zona periorbitaria, siendo ésta una manifestación fisiológica que puede mantenerse alrededor de una semana. El periodo de reposo laboral habitualmente no supera las tres semanas.

Para el control de la reducción y fijación de la fractura se efectuó una radiografía panorámica y una fronto naso placa y comparativa de órbita a los 7 días sucesivos donde se observó correcta proyección del piso de la órbita.

\section{Discusión}

En los últimos años ha habido un considerable avance de los biomateriales disponibles para la reconstrucción de suelo de la órbita y al mismo tiempo, la experiencia quirúrgica y observación clínica hizo posible seleccionar el material más fiable y adecuado para la aplicación clínica.

Los injertos óseos fueron ampliamente utilizados, donde los sitios donantes fueron principalmente calota craniana y cresta iliaca; según Ochs en el
2004 demostraba que la morbilidad aumentaba y los riesgos de extraer injertos de calota o un nuevo acceso quirúrgico en el caso de la cresta iliaca han estimulado la búsqueda de nuevos elementos reconstructivos. Desde entonces, diferentes materiales aloplásticos han sido utilizados, donde la malla de titanio se ha evaluado como un excelente medio reconstructivo. Sin embargo, existe una línea de trabajo encaminada por Jacquiéry que estipula, en casos de trauma complejo de órbita, el uso de más de un material, conjugando los injertos óseos y los materiales aloplásticos como malla de titanio 4 .

Por su parte la Dra. Rodrigues Cunha Colombo afirma que las mallas de titanio al ser fijadas por tornillos, impiden el movimiento y la migración de los cabos de fractura, reduciendo así el riesgo de extrusión y la necesidad de extracción, que se ve obstaculizada por la incorporación de tejido fibroso y el hueso que lo rodea.

En el 2004 el Dr. Martínez Villalobos Castillo S. manifestaba que los biomateriales reabsorvibles eran alternativas menos seguras y contrastadas pero ya con un futuro esperanzador que se abría camino poco a poco en indicaciones como la cirugía ortognática y en la traumatológica del tercio medio facial del adulto. En la actualidad, 
es objeto de estudio de muchos investigadores y cirujanos que se encuentran empleando en su campo $^{10}$.

Por lo tanto, existen distintos materiales que se pueden utilizar con el fin de reconstruir el suelo de la órbita. La elección se asocia con la disponibilidad de material, la relación costo-beneficio de cada uno y la planificación previa de cada caso.

\section{Conclusión}

El trauma es una causa importante de morbilidad y mortalidad de hoy. Entre los distintos tipos de trauma el problema radica en el impacto físico, emocional y la posibilidad de daño permanente. Existe una alta tasa de lesiones traumáticas de la cara en comparación con otras áreas del cuerpo. Esto se debe a la exposición grande y poca protección en la región facial.

Los traumas del tercio medio facial representan una parte importante del trauma maxilofacial donde la fractura de órbita representa del $10 \%$ al $25 \%$ de los casos. 8

El concepto más importante en el tratamiento de estas fracturas orbitocigomáticas es su correcta reducción, exponiéndose todos los focos de fractura de forma simultánea. No existe unanimidad en la literatura sobre qué fracturas podemos tratar con procedimientos simples de reducción y qué otras van a necesitar tratamiento quirúrgicos más agresivos, con desperiostización amplia y reducción abierta. De todas formas, en la literatura médica se impone progresivamente la filosofía de proporcionar una reducción anatómica (más o menos invasiva según el caso) y una osteosíntesis adecuada (suficientemente estable) para garantizar una "restitutio ad integrum" en forma y función, en el menor periodo de tiempo posible.

\section{Bibliografía}

I. Araújo A, Araújo, MM, Cavalieri M, Costa Silva Pereira C, Andrade de Oliva M, Amaral Costa D. Acesso transconjuntival para fraturas do complexo zigomático-orbitário. Cir. Traumatol. BucoMaxilo-Fac. Camaragibe: 6 (4); p. 39 - 48, 2006.

2. Almeida Parra F, Picón Medina M, MartínezVillalobos,S, García-Rozado González A. Fracturas orbitomalares. Cap 15. Protocolos Clínicos de la Sociedad Española de Cirugía Oral y Maxilofacial.

3. Ellis III E, Tan Y. Assessment of internal orbital reconstructions for pure blowout fractures: cranial bone grafts versus titanium mesh. 2003. J Oral Maxillofac Surg; 61: p 442-453.

4. OLATE S, ALISTER HJP, CHAVES NETTO DDMH, JAIMES M, MAZZONETTO R. Reconstrucción de piso de órbita con malla de titanio en trauma de tercio medio facial. Int. J. Odontostomat.2008; 2(2):163-I70.

5. Ellis III E y Zide MF. Abordajes Quirúrgicos del Macizo Facial. 2da ed. Colombia: AMOLCA; 2008.

6. Montilla OS. Tratado de Cirugía Oral y Maxilofacial. Introducción básica a la enseñanza. Venezuela: AMOLCA; 2007.

7. Guralnick WC y cols. Tratado de Cirugía Oral. España: SALVAT; 197I.

8. Roth FS, Koshy JC, Goldberg JS, Soparkar C. Pearls of Orbital Trauma Management. Semin Plast Surg. 2010; 24 (4): 398-410.

9. Rodrigues Cunha Colombo L, Reis Calderoni $D$, Toresan Rosim E, Passeri LA. Biomateriais para reconstrução da órbita. Rev. Bras. Cir. Plást. (Impr.) [online]. 20I I; 26 (2) p. 337-342.

10. Martínez Villalobos Castillo S. Osteosíntesis maxilofacial con titanio. Rev Esp Cirug Oral y Maxilofac. Madrid. 2004; 26 (6)

II. Chamorro M, Gómez E. Fracturas Orbitocigomáticas. En: Martínez - Villalobos S: Osteosíntesis Craneo maxilofacial. Cap.6: 8I. Ed. Ergon. Madrid, 2002.

12. Kovacs AF, Ghahremani M. Minimization of zygomatic complex fracture treatment. Int J Oral Maxillofac Surg.200 I; 30 (5): 380-3.

13. Ellis $E$, Reddy $L$. Status of the internal orbit after reduction of zygomaticomaxillary complex fractures. J. Oral Maxillofac. Surg., 62:275-83, 2004.

14. Erdmann D, Follmar KE, Debruijn M y cols. Un análisis retrospectivo de causas de fracturas faciales. Ann Plast Surg. 2008; 60:398-403

15. Mellema PA, Dewan MA, Lee MS, SD Smith, Harrison $\mathrm{R}$. Incidencia de lesiones oculares en las fracturas orbitarias visualmente asintomáticos. Ophthal Plast Surg Reconstr. 2009; 25: 306-308.

16. Bater MC, Ramchandani PL, Brennan PA. Posttraumatic eye observation. Br. J. Oral Maxillofac. Surg. 2005; 43: 410-6. 